\title{
QUALIDADE DE VIDA E ESTRATÉGIAS DE ENFRENTAMENTO DE PACIENTES EM TRATAMENTO ONCOLÓGICO
}

\author{
Fernanda Ottati ${ }^{1 *}$, Mariana Pinhatari Souza Campos ${ }^{1 * *}$ \\ ${ }^{1}$ Universidade São Francisco - Brasil
}

Recibido, diciembre 17/2013

Concepto de evaluación, abril 24/2014

Aceptado, mayo 28/2014
Referencia: Ottati, F. \& Souza Campos, M.P. (2014). Qualidade de vida e estratégias de enfrentamento de pacientes em tratamento oncológico. Acta Colombiana de Psicología, 17 (2), pp. 103-111. DOI:10.14718/ACP.2014.17.2.11

Resumo

O objetivo deste estudo foi verificar a relação entre percepção da qualidade de vida e as estratégias de enfrentamento em pessoas em tratamento quimioterápico. Participaram 42 pacientes, $22(52,4 \%)$ mulheres e 20 (47,6\%) homens, com idade média de 57 anos $(M=57,07 ; \mathrm{DP}=13,269)$. Os instrumentos utilizados foram a Escala de modos de enfrentamento de problemas - EMEP e WHOQOL-Bref. Os resultados indicam que os pacientes que estão em fase inicial do tratamento fazem maior uso de estratégias que modificam ou alteram o evento estressor (o tratamento oncológico) com o objetivo de controlar ou lidar melhor com a situação, além de se envolverem mais com práticas religiosas ou pensamentos fantasiosos para auxiliar o processo de enfrentamento. Os dados reafirmam a importância da relação entre os construtos, que, quando utilizados de forma eficaz promovem resultados significativos na vida do paciente e na sua adaptação frente à doença e ao tratamento.

Palavras chave: qualidade de vida, psiconcología, câncer, coping, quimioterapia.

\section{CALIDAD DE VIDA Y ESTRATEGIAS DE AFRONTAMIENTO EN EL TRATAMIENTO DE PACIENTES ONCOLÓGICOS}

\begin{abstract}
Resumen
El objetivo de este estudio fue investigar la relación entre la percepción de calidad de vida y las estrategias de afrontamiento en personas que reciben quimioterapia. Participaron 42 pacientes, 22 mujeres $(52,4 \%)$ y 20 hombres (47.6\%), con edad media de 57 años $(\mathrm{M}=57.07, \mathrm{SD}=13.269)$. Los instrumentos utilizados fueron la Escala de estrategias de afrontamiento de problemas [EMEPpor sus siglas en portugués] y el WHOQOL-Bref. Los resultados indican que los pacientes en la fase inicial del tratamiento hacen un mayor uso de estrategias que modifican o alteran el factor estresante (tratamiento del cáncer) con el fin de controlar o enfrentar mejor la situación, y tener una mayor participación en las prácticas religiosas o imaginarias que favorecen el proceso de afrontamiento de pensamientos. Los datos reafirman la importancia de la relación entre constructos, que utilizados de manera efectiva, promueven resultados significativos en la vida del paciente y su adaptación a la enfermedad y al tratamiento.

Palabras clave: calidad de vida, psicooncología, cáncer, afrontamiento; quimioterapia.
\end{abstract}

\section{QUALITY OF LIFE AND COPING STRATEGIES IN THE TREATMENT OF ONCOLOGIC PATIENTS}

\begin{abstract}
The aim of this study was to determine the relationship between perception of quality of life and coping strategies in people undergoing chemotherapy. Participants were 42 patients, 22 (52.4\%) women and 20 (47.6\%) men, mean age 57 years old $(M=57.07 ; S D=13,269)$. The instruments used were the Problem Coping Strategies Scale- [EMEP, for its Portuguese acronym] and the WHO Quality of Life-BREF (WHOQOL-BREF). Results indicate that patients who are in the initial phase of treatment make greater use of strategies that modify or alter the stressor (oncologic treatment) in order to control or cope better with the situation and be more involved in religious or imaginary practices that help in the process of coping with thoughts. Data affirm the importance of the relationship between the constructs, which, when used effectively, promote significant results in the patient's life and their adaptation to the disease and treatment.

Key words: quality of life, psychosocial oncology, cancer, coping, chemotherapy.
\end{abstract}

\footnotetext{
* Psicóloga, Doutora em Psicologia. Docente do Curso de Psicologia da Universidade São Francisco - Brasil -SP. fernanda_itb@yahoo.com.br

** Mariana Pinhatari Souza Campos. Rua Caribe, 139 - Jd, Adélia, Amparo/SP, Brasil. CEP: 13904-220. ma.pinhatari@hotmail.com
} 


\section{INTRODUÇÃO}

Segundo as últimas estimativas divulgadas pelo Instituto Nacional de Câncer José de Alencar Gomes da Silva (INCA) o câncer pode ter acometido cerca de 520 mil brasileiros (casos) nos anos de 2012 e 2013 . Ele está presente na história da humanidade desde a Grécia Antiga, e desde então está vinculado à morte $\mathrm{e}$ a algo ameaçador, se tornando temido pela população (Sebben, 2007; Carvalho et al., 2008).

Com os avanços na atuação da Psicologia Hospitalar alguns fatores emocionais e psicológicos ligados ao diagnóstico e tratamento do câncer passaram a ser observados, como a associação à morte iminente, o medo da dor, do sofrimento, da desintegração moral/social e da solidão, dentre outros. Diante disso, e da eficácia advinda da atuação do psicólogo, esta prática passou a ser reconhecida, emergindo assim a área da Psiconcologia (Carvalho, 1994; Carvalho et al., 2008).

Esta pode ser descrita como a interconexão entre Psicologia e Oncologia e visa auxiliar o paciente a se adaptar (emocional e socialmente) durante o tratamento, se tornando elemento essencial ao desenvolvimento e manutenção da doença. É uma ampla área de atuação e se preocupa em ajudar o paciente a lidar com as reações que a doença causa e também com os estigmas existentes em torno da mesma (Carvalho, 1994; Sebben, 2007; Canciam, 2011).

Sendo assim, promover qualidade de vida se torna foco na atuação dos profissionais, de forma que propiciem ao paciente uma melhora em sua qualidade de vida (QV), garantindo assim, uma ação ativa em seu próprio tratamento, pois eles sofrem grande impacto sobre sua vida, seus hábitos e rotina, exigindo uma grande adaptação psicológica a esta nova condição (Carvalho et al., 2008; Souza, 2009).

Não há um consenso sobre a definição de qualidade de vida, e esta dificuldade se dá por ser um conceito subjetivo, onde cada um a percebe de uma maneira muito particular, variando de acordo com valores, princípios, cultura, classe social, e até mesmo a época em que se está vivendo (Oliveira, Pereira, Pontes, Fialho \& Moreira, 2007 citados por Souza, 2009). Dentre os conceitos existentes na literatura, o mais abrangente é definido pela Organização Mundial de Saúde (OMS): "a percepção do indivíduo sobre sua posição na vida, no contexto da cultura e dos sistemas de valores nos quais ele vive, e em relação a seus objetivos, expectativas, padrões e preocupações" (Fleck et al, 2008, p. 25).

A QV, assim como muitos outros conceitos, sofre algumas influências de mediadores psicológicos, como nomeados por Pestana, Estevens e Conboy (2007) e Costa Neto e Araújo (2008), e dentre eles encontra-se o coping ou enfrentamento (denominação brasileira). Gimenes (1998),
Seidl, Tróccoli e Zannon (2001) e Souza (2009) definem enfrentamento como sendo os esforços que o indivíduo dedica para modificar a situação estressora, com o objetivo de controla-la ou de resolver problemas. Os autores explicam que ao utilizar estas estratégias a pessoa faz uso de recursos cognitivos e comportamentais para lidar com os eventos estressores. Considerando que o evento estressor seja o câncer, os esforços são direcionados a cada fase da doença, pois cada uma delas exige novas adaptações.

Sob esta perspectiva, Lorencetti e Simonetti (2005), Souza (2009) e Seidl, Tróccoli e Zannon (2001) colocam que estas estratégias podem ser distribuídas em duas funções, as estratégias com foco na emoção e as estratégias com foco no problema. A primeira tem caráter defensivo e normalmente evita-se o contato consciente com a realidade, envolve ações paliativas em relação à dificuldade, com o intuito de amenizar o impacto emocional ocasionado pelo problema. Já quando o foco é no problema, as estratégias são direcionadas para a realidade, onde ocorre uma aproximação ao evento estressor com o objetivo de diminuir ou anular o seu impacto e melhorar sua relação com o ambiente, por meio de soluções, ações e/ou estratégias traçadas a partir desse contato. Os autores ainda relatam que dificilmente um indivíduo faz uso único de uma ou outra estratégia, mas sim de uma mistura de ambas, que podem estar sendo usadas em maior ou menor quantidade.

Pelo fato de cada fase do tratamento oncológico se configurar como um evento estressor, as estratégias de enfrentamento focalizadas no problema são as mais utilizadas, a forma como o paciente lida com o problema gera efeito sob sua adaptação a nova fase e também, por meio de seu uso é possível identificar um nível satisfatório em QV. Diante disso, práticas terapêuticas devem ser estruturadas com o intuito de minimizar o impacto causado pela doença e pelo tratamento, sendo então direcionadas para auxiliar na adaptação do indivíduo a nova realidade e ao uso favorável das estratégias de enfrentamento (Santana, Zanin \& Maniglia, 2008; Souza, 2009; Alegrance, Souza \& Mazzei, 2010).

Devido a sua importância, a relação entre QV e estratégias de enfrentamento vem ganhado destaque no meio científico, a preocupação com esses pacientes, assim como um novo olhar sobre as estratégias terapêuticas direcionadas a eles estão aumentando, pois estas podem levar a uma melhora significativa na qualidade de vida (Souza \& Araújo, 2010). Um exemplo disso, é o trabalho de Alegrance, Souza e Mazzei (2010), que buscou avaliar as estratégias de enfrentamento e a percepção de qualidade de vida de pacientes com ou sem linfedema após câncer de mama com o objetivo de identificar quais estratégias de enfrentamento estavam sendo utilizadas frente à doença, qual a percepção 
de qualidade de vida e verificar o impacto desta complicação nos fatores biopsicossociais de 32 mulheres. Do total da amostra, 39,03\% apresentaram linfedema após o câncer de mama, mas isso não interferiu significativamente na percepção de qualidade de vida.

As correlações entre os construtos qualidade de vida e estratégias de enfrentamento entre as pacientes com ou sem linfedema apontam que para as duas populações a qualidade de vida geral é boa e os aspectos biopsicossociais comprometidos são semelhantes. Já para as estratégias de enfrentamento o observado foi que as estratégias ativas e positivas como reavaliação, resolução de problemas, suporte social e auto controle foram utilizadas por ambas as populações, o que confirma que o uso destas estratégias está diretamente vinculado a boa adaptação biopsicossocial (Alegrance et al., 2010).

Souza e Araújo (2010), por meio de uma intervenção em grupo de pacientes oncológicos em início de quimioterapia, buscavam promover habilidades adaptativas, enfrentamento e consequentemente uma melhora na $\mathrm{QV}$ do grupo. $\mathrm{O}$ estudo foi constituído de 3 fases (Fase 1 - Linha de base, Fase 2 - Intervenção em grupo e Fase 3 - Follow up), nas fases 1 e 3 foram avaliados a percepção da qualidade de vida e estratégias de enfrentamento utilizadas. Os dados obtidos apontaram que após a intervenção as estratégias de enfrentamento do grupo apresentaram uma melhora significativa em todos os fatores, bem como um aumento na percepção de qualidade de vida.

Roque e Forones (2006) também se preocuparam em avaliar a percepção de QV em pacientes com câncer, o estudo foi desenvolvido no Ambulatório de Oncologia da Clínica da Universidade Federal de São Paulo, com pacientes em tratamento quimioterápico adjuvante ou paliativo, com duração de seis ciclos. O construto foi avaliado antes do início do primeiro, terceiro e sexto ciclos quimioterápicos, e os resultados indicaram que as dimensões físicas e psicológicas se alteram em média, entre 63 a $65 \%$ dos participantes em todos os ciclos de tratamento. Já as dimensões sociais e ambientais se encontravam abaixo do escore padrão, respectivamente em $44,8 \%$ e $47,5 \%$ dos participantes. Os domínios físicos e sociais apresentaram uma queda significativa na aplicação que ocorreu antes do inicio do sexto ciclo, concluindo que a QV diminui quando os pacientes oncológicos dão início ao tratamento quimioterápico, principalmente em relação às dimensões físicas e psicológicas.

O trabalho de Huguet, Morais, Osis, Pinto-Neto e Gurgel (2009) também é relevante, e por meio da avaliação da percepção de QV em mulheres que já haviam passado por tratamento de câncer de mama há pelo menos um ano no Centro de Atenção Integral à Saúde da Mulher da UNI-
CAMP perceberam, em análise geral, que as participantes do estudo apresentaram uma boa qualidade de vida. Os autores apontam que isso pode ser resultado do auxílio multidisciplinar oferecido pela instituição, além das variáveis socioeconômicas, de escolaridade, estado civil e conservação mamária das pacientes.

Santana, Zanin e Maniglia (2008), verificaram a condição das estratégias de enfrentamento em 22 pacientes oncológicos de cabeça e pescoço. Os dados coletados indicaram que fatores busca por suporte social $(\mathrm{M}=2,62 \mathrm{e} \mathrm{DP}=0,80) \mathrm{e}$ enfrentamento focalizado na emoção $(\mathrm{M}=2,08$ e $\mathrm{DP}=0,63)$ foram os menos utilizados pelos pacientes, enquanto que o enfrentamento focalizado no problema e a busca por práticas religiosas/pensamentos fantasiosos apresentaram correlação positiva $(\mathrm{r}=0,579, \mathrm{p}<0,05)$. Quando os resultados são comparados com os escores padrão da escala (EMEP), observa-se que eles se encontram dentro do esperado, ficando fora do padrão $(\mathrm{M}=3,62 \mathrm{e} \mathrm{DP}=0,67)$ somente o fator busca por suporte social $(\mathrm{M}=2,62$ e $\mathrm{DP}=0,80)$. Em relação ao menor escore obtido entre os fatores, o enfrentamento focalizado na emoção foi o que se destacou, da mesma forma que encontrado no estudo de validade da escala.

Ainda sobre o tema, Sebben (2007) aponta que a avaliação destes construtos é de grande interesse para os profissionais de saúde, pelo fato de somar e ampliar o conhecimento em sua prática, gerando benefícios a todos os envolvidos no tratamento oncológico (profissional, equipe, família e paciente). $\mathrm{O}$ autor ainda explana que em relação à psicologia, estes conhecimentos auxiliam no entendimento dos aspectos psicológicos envoltos da doença, e a partir disso, sua atuação favorece a transição do paciente e sua família pela enfermidade, auxiliando na melhora do equilíbrio emocional, do relacionamento interpessoal e, consequentemente, leva a um tratamento mais efetivo.

Baratto et al. (2011) complementam que os psicólogos buscam oferecer suporte para que o paciente lide de uma forma mais efetiva com as questões emergidas pelo câncer e seu tratamento, e devido aos benefícios que pode gerar, se torna prática essencial no serviço oferecido ao paciente e familiares. Deste modo, estudos relacionados à percepção de qualidade de vida e as estratégias de enfrentamento focalizadas no problema, além de contribuir para o avanço do conhecimento científico, fornecem material de trabalho não só para psicólogos, mas para todos os profissionais que lidam com pacientes oncológicos, norteando sua terapêutica e, consequentemente, gerando algum efeito positivo sob a vida dos pacientes e seu bem estar (Souza, 2009).

Diante de tudo o que foi colocado, o objetivo deste estudo foi verificar a relação entre a percepção da qualidade de vida e as estratégias de enfrentamento de pessoas em 
tratamento oncológico, bem como identificar as diferenças em relação à idade e a fase do tratamento.

\section{MÉTODO}

\section{Participantes}

Apesquisa foi realizada com 42 pacientes de uma clínica especializada no atendimento de pacientes oncológicos, que estavam em tratamento quimioterápico, sendo 52,4\% (22) do sexo feminino e 47,6\% (20) do sexo masculino com idade entre 27 e 84 anos $(M=57,07$ e $D P=13,269)$. Dos 42 pacientes entrevistados $71,4 \%(\mathrm{~N}=30)$ estavam passando pela primeira vez por um tratamento oncológico e, $61,9 \%$ $(\mathrm{N}=26)$ não se encontravam em fase inicial do tratamento. O tempo de tratamento variou de uma semana a nove anos, sendo que primeira semana $(n=7,16,7 \%)$, seis meses $(n=$ $5,11,9 \%)$ e um ano $(n=6,14,3 \%)$ apareceram com maior frequência.

\section{Instrumentos}

Escala de modos de enfrentamento de problemas (EMEP). Foi adaptada ao português por Gimenes e Queiroz (1997), e posteriormente, validada por Seidl, Tróccoli e Zannon (2001), versão utilizada neste trabalho. É uma escala auto aplicável que avalia o enfrentamento, considerando quais estratégias (cognitivas e/ou comportamentais) o paciente utiliza para enfrentar a doença (evento estressor). Ela é composta por 45 itens em escala tipo Likert de 5 pontos, que são distribuídos em quatro fatores, sendo eles: Enfrentamento focalizado no problema - essas estratégias tem por objetivo controlar ou lidar com a situação, normalmente são o meio de aproximação em relação ao evento estressor; Enfrentamento focalizado na emoção - Avaliam as estratégias da pessoa em adequar sua resposta emocional ao evento estressor, podendo representar atitudes de alienação ou paliativas em relação ao problema; Busca de práticas religiosas/pensamento fantasioso - Envolvem as estratégias como comportamentos religiosos ou pensamentos que possam auxiliar no enfrentamento; e Busca de suporte social-mensura as estratégias usadas para auxiliar a pessoa a enfrentar o problema advindas da procura por apoio instrumental, emocional ou informação.

O instrumento foi escolhido por ser uma escala validada para a população brasileira, e também por ser apropriado para pacientes com patologias crônicas. Seidl, Tróccoli e Zannon (2001), consideram que a capacidade psicométrica do instrumento é positiva, e foi obtida a partir de uma amostra final de 409 adultos de diferentes instituições de ensino e locais de trabalho onde o evento estressor era uma situação atual e de pessoas com doenças crônicas de um hospital público e de um serviço especializado e o evento estressor era sua situação atual de saúde.

WHOQOL-Bref. É um instrumento da Organização Mundial de Saúde (OMS), validado para a população brasileira por Fleck (2008) para avaliação da percepção da qualidade de vida, considerando que a mesma é caracterizada por ser um construto subjetivo, multidimensional e composto por dimensões positivas e negativas. Esta versão é composta por 26 questões tipo Likert (escala de 5 pontos ) que são subdivididas em 04 domínios: Físico - que mede a percepção do sujeito em relação a sua condição física; Psicológico - que avalia a percepção da pessoa sobre sua condição afetiva e cognitiva; Relações Sociais - mensura a percepção da pessoa sobre seus relacionamentos sociais e também dos papéis sociais tomados em sua vida; e Meio Ambiente - que afere a percepção da pessoa em relação aos aspectos diversos relacionados ao meio em que vive. O instrumento é considerado confiável, fidedigno e válido segundo as propriedades psicométricas.

\section{Procedimento}

Inicialmente foi solicitada a autorização da clínica e após o projeto foi encaminhado ao Comitê de Ética em Pesquisa da USF (protocolo CAAE: 09543812.1.0000.5514). A partir da aprovação, a clínica foi contatada para agendar o início da coleta que teve duração de três meses e ocorreu da seguinte forma, o contato inicial com cada paciente foi realizado na recepção da clínica, este era convidado a uma conversa com a pesquisadora em sala reservada, onde os objetivos da pesquisa eram expostos e havendo a concordância em participar da mesma a assinatura do Termo de Consentimento Livre e Esclarecido era realizada.

Com o consentimento para a realização da pesquisa dava-se início a aplicação dos instrumentos, sendo primeiro o EMEP e posteriormente WHOQOL-Bref. A aplicação variou de 20 minutos a $1 \mathrm{hr} 30 \mathrm{~min}$ de acordo com cada paciente, que aproveitavam o momento para compartilhar sua história e sua vivência frente ao câncer e ao tratamento. Além disso, a maioria dos sujeitos solicitou a leitura do material pela pesquisadora (por motivos como falta de óculos, dificuldade de leitura, dentre outros), que preenchia item a item as respostas verbalizadas. As entrevistas ocorreram de forma individual nas dependências da clínica em momento anterior ao tratamento e dos 44 pacientes abordados apenas 2 se recusaram a participar da pesquisa.

\section{RESULTADOS}

Para atender os objetivos deste trabalho foram realizadas análises da correlação de Pearson e o teste $t$ de Student, por meio do programa estatístico SPSS. Foram calculadas as 
Tabela 1.

Diferença de média em relação a estar em fase inicial do tratamento quimioterápico

\begin{tabular}{|c|c|c|c|c|c|c|}
\hline & $\begin{array}{l}\text { Fase inicial do } \\
\text { tratamento? }\end{array}$ & $N$ & Média & $D P$ & $t$ & $p$ \\
\hline \multirow[t]{2}{*}{ Enfretamento foco problema } & Sim & 16 & 4,03 & 0,413 & 2,778 & 0,008 \\
\hline & Não & 26 & 3,56 & 0,593 & & \\
\hline \multirow[t]{2}{*}{ Enfretamento foco emoção } & Sim & 16 & 1,76 & 0,638 & $-0,482$ & 0,633 \\
\hline & Não & 26 & 1,84 & 0,419 & & \\
\hline \multirow[t]{2}{*}{ Religioso pensamento fantástico } & Sim & 16 & 4,08 & 0,772 & 2,175 & 0,036 \\
\hline & Não & 26 & 3,47 & 0,943 & & \\
\hline \multirow[t]{2}{*}{ Busca suporte social } & Sim & 16 & 3,50 & 0,897 & 1,881 & 0,067 \\
\hline & Não & 26 & 2,95 & 0,923 & & \\
\hline \multirow[t]{2}{*}{ Domínio físico } & Sim & 16 & 3,83 & 0,728 & 0,963 & 0,341 \\
\hline & Não & 26 & 3,62 & 0,656 & & \\
\hline \multirow[t]{2}{*}{ Domínio psicológico } & Sim & 16 & 4,00 & 0,505 & 2,014 & 0,051 \\
\hline & Não & 26 & 3,58 & 0,724 & & \\
\hline \multirow[t]{2}{*}{ Domínio relações sociais } & Sim & 16 & 3,85 & 0,515 & 0,291 & 0,773 \\
\hline & Não & 26 & 3,79 & 0,706 & & \\
\hline \multirow[t]{2}{*}{ Domínio meio ambiente } & Sim & 16 & 3,84 & 0,533 & 0,165 & 0,870 \\
\hline & Não & 26 & 3,81 & 0,487 & & \\
\hline \multirow[t]{2}{*}{ QV total } & Sim & 16 & 3,68 & 0,655 & 0,867 & 0,391 \\
\hline & Não & 26 & 3,46 & 0,904 & & \\
\hline
\end{tabular}

Tabela 2.

Diferença de média em relação a grupo extremos de idade.

\begin{tabular}{|c|c|c|c|c|c|c|}
\hline & Idade & $N$ & Média & $D P$ & $t$ & $p$ \\
\hline \multirow[t]{2}{*}{ Enfretamento focoproblema } & Até 47,75 anos & 10 & 4,00 & 0,363 & 2,754 & 0,013 \\
\hline & Mais de 65,5 anos & 10 & 3,45 & 0,508 & & \\
\hline \multirow[t]{2}{*}{ Enfretamento foco emoção } & Até 47,75 anos & 10 & 1,88 & 0,638 & 1,225 & 0,236 \\
\hline & Mais de 65,5 anos & 10 & 1,61 & 0,299 & & \\
\hline \multirow[t]{2}{*}{ Religioso pensamento fantástico } & Até 47,75 anos & 10 & 4,04 & 0,858 & 2,042 & 0,056 \\
\hline & Mais de 65,5 anos & 10 & 3,18 & 1,021 & & \\
\hline \multirow[t]{2}{*}{ Busca suporte social } & Até 47,75 anos & 10 & 3,56 & 0,556 & 2,049 & 0,055 \\
\hline & Mais de 65,5 anos & 10 & 2,80 & 1,032 & & \\
\hline \multirow[t]{2}{*}{ Domínio físico } & Até 47,75 anos & 10 & 3,61 & 0,747 & 0,229 & 0,822 \\
\hline & Mais de 65,5 anos & 10 & 3,54 & 0,645 & & \\
\hline \multirow[t]{2}{*}{ Domínio psicológico } & Até 47,75 anos & 10 & 3,61 & 0,765 & $-0,438$ & 0,667 \\
\hline & Mais de 65,5 anos & 10 & 3,75 & 0,583 & & \\
\hline \multirow[t]{2}{*}{ Domínio relações sociais } & Até 47,75 anos & 10 & 3,90 & 0,770 & 0,383 & 0,706 \\
\hline & Mais de 65,5 anos & 10 & 3,76 & 0,786 & & \\
\hline \multirow[t]{2}{*}{ Domínio meio ambiente } & Até 47,75 anos & 10 & 3,66 & 0,520 & $-0,827$ & 0,419 \\
\hline & Mais de 65,5 anos & 10 & 3,86 & 0,560 & & \\
\hline \multirow[t]{2}{*}{ QV total } & Até 47,75 anos & 10 & 3,70 & 0,632 & 1,134 & 0,272 \\
\hline & Mais de 65,5 anos & 10 & 3,30 & 0,918 & & \\
\hline
\end{tabular}


Tabela 3.

Correlação de Pearson entre EMEP e WHOQOL-Bref

\begin{tabular}{lccccc}
\hline & $\begin{array}{c}\text { Dominio } \\
\text { fisico }\end{array}$ & $\begin{array}{c}\text { Domínio } \\
\text { psicológico }\end{array}$ & $\begin{array}{c}\text { Domínio relações } \\
\text { sociais }\end{array}$ & $\begin{array}{c}\text { Domínio meio } \\
\text { ambiente }\end{array}$ & $\begin{array}{c}Q V \\
\text { total }\end{array}$ \\
\hline Enfrentamento foco problema & 0,059 & 0,254 & 0,190 & 0,158 & 0,212 \\
Enfrentamento foco emoção & $-0,214$ & $\mathbf{- 0 , 4 2 4 ^ { * * }}$ & $-0,234$ & $-0,036$ & $-0,050$ \\
Religioso pensamento fantástico & $-0,005$ & $-0,160$ & $-0,105$ & 0,034 & 0,108 \\
Busca suporte social & 0,193 & 0,181 & $\mathbf{0 , 3 6 4}$ & 0,227 & $\mathbf{0 , 3 3 8}^{*}$ \\
\hline * correlação significativa 0,005 & & & &
\end{tabular}

médias ponderadas para cada um dos domínios e fatores dos instrumentos, obtida pela soma dos itens que compõe cada fator dividido pelo número de itens. Dessa forma, a pontuação mínima será sempre 1 e a pontuação máxima será sempre 5. As diferenças na percepção de qualidade de vida e modos de enfrentamento de problemas em relação à fase do tratamento oncológico ser inicial ou não, foi verificada por meio do teste t de Student. Os dados estão na tabela 1.

Em relação ao tratamento oncológico estar ocorrendo pela primeira vez ou não, foram encontrados resultados estatisticamente significativos apenas em dois fatores da EMEP e um domínio do WHOQOL-Bref. Os dados apontaram que os pacientes que estão em fase inicial do tratamento fazem maior uso das estratégias de enfrentamento focalizadas no problema e da busca por práticas religiosas ou pensamentos fantasiosos. Além disso, os dados mostram que os pacientes que estão em fase inicial do tratamento fazem maior uso do domínio psicológico.

Para verificação de possíveis diferenças nas estratégias de enfrentamento e percepção de qualidade de vida, em relação à idade dos participantes, primeiramente foi feita a divisão dos grupos de idade, a partir do quartil, de forma que os dados foram agrupados em quatro grupos de idade, sendo eles: idade até 47,75 anos $(f=10,23,8 \%)$, entre 47,46 e 58 anos $(f=13,31 \%)$, entre 58,1 e 65,5 $\operatorname{anos}(f=9,21,4 \%)$, e mais de 65,5 anos $(f=10,23,8 \%)$. Diante disso, foi observado, a partir deste agrupamento, que a maior parte da amostra encontra-se com idade entre 47,76 e 58 anos (31\%), enquanto que a faixa de idade de 58,1 a 65,5 anos apresentou a menor frequência nesta população $(21,4 \%)$.

Ainda em relação a variável idade, foi realizada a prova $t$ de student entre os dois grupos extremos, ou seja, os mais novos e mais velhos da amostra. Os dados estão na tabela 2.

Foram encontrados resultados significativos em três fatores da EMEP. Desta forma, pode-se observar que pacientes mais novos fazem uso maior das estratégias de enfrentamento focalizadas no problema $(M=4,00 ; p=0,013)$, da busca por práticas religiosas ou pensamento fantasioso $(M=3,56 ; p=0,056)$ e da busca por suporte social $(\mathrm{M}=3,56$; $\mathrm{p}=0,055)$.

Por fim, a última análise realizada foi verificar a relação entre qualidade de vida e modo de enfrentamento de problemas por meio da correlação de Pearson que apontou correlações negativas e positivas, conforme tabela 3 .

Foi encontrada correlação negativa entre o domínio psicológico e a estratégia de enfrentamento focalizada na emoção $(r=-0,424)$. Além disso, houve correlação positiva $(r=0,364)$ entre a percepção do sujeito sobre seus relacionamentos sociais e de seus papeis sociais (domínio relações sociais) e as estratégias utilizadas para auxiliá-lo a enfrentar o evento estressor, sejam elas advindas de um apoio instrumental, emocional ou de informações (busca por suporte social). A busca por suporte social, também apresentou correlação positiva com o domínio qualidade de vida total $(r=0,338)$,

\section{DISCUSSÃO}

Após a análise dos resultados obtidos é possível perceber que, os pacientes que estão em fase inicial do tratamento fazem maior uso de estratégias que modificam ou alteram o evento estressor (o tratamento oncológico) com o objetivo de controlar ou lidar melhor com a situação (estratégias de enfrentamento focalizadas no problema), além de se envolverem mais com práticas religiosas ou pensamentos fantasiosos para auxiliar o processo de enfrentamento (busca por práticas religiosas ou pensamentos fantasiosos), como também apresentaram uma percepção maior sobre sua condição afetiva e/ou cognitiva (domínio psicológico).

Em relação a variável idade os pacientes mais novos, quando comparados aos mais velhos, apresentaram mais comportamentos relacionados ao controle ou modificação da 
situação estressora (enfrentamento focalizado no problema) usando estratégias que possam auxiliar no enfrentamento, sejam elas relacionadas à religião ou a ideações fantasiosas (busca por práticas religiosas ou pensamento fantasioso), ou advindas da procura de apoio instrumental, emocional ou de informações sobre a situação (busca por suporte social).

Comparando esses (dois grupos extremos da amostra deste trabalho - Até 47,75 anos e Mais de 65,5 anos) com os obtidos no estudo de Santana, Zanin e Maniglia (2008), é possível afirmar que a diferença da média de idade dos participantes é uma variável importante a ser considerada para o entendimento das estratégias que são utilizadas. Os autores verificaram a condição das estratégias de enfrentamento e os resultados indicaram que a busca por suporte social e o enfrentamento focalizado na emoção foram os menos utilizados pelos pacientes, enquanto que o enfrentamento focalizado no problema apresentou o maior escore.

Santana et al (2008) comentam que estudos relacionados às estratégias de enfrentamento focalizadas no problema em pacientes oncológicos apresentam um panorama sobre o funcionamento das estratégias utilizadas por eles, além de levantar demandas que podem orientar o trabalho dos profissionais de saúde que atuam com esta população, principalmente os psicólogos.

A relação entre qualidade de vida e modo de enfrentamento de problemas indicou uma correlação negativa entre o domínio psicológico e a estratégia de enfrentamento focalizada na emoção $(r=-0,424)$ o que pode ser explicado pelo fato de o domínio psicológico estar relacionado à percepção do indivíduo tanto sobre sua condição afetiva como sobre sua condição cognitiva enquanto que o enfrentamento focalizado na emoção está vinculado à resposta emocional do sujeito, podendo esta ser relacionada a atitudes de alienação ou paliativas em relação à situação estressora.

Já a correlação positiva $(r=0,364)$ entre a percepção do sujeito sobre seus relacionamentos sociais e de seus papeis sociais (domínio relações sociais) e as estratégias utilizadas para auxiliá-lo a enfrentar o evento estressor, sejam elas advindas de um apoio instrumental, emocional ou de informações (busca por suporte social), indicaram que estes fatores são complementares, auxiliando, através do meio social, na passagem pelo evento estressor. A busca por suporte social, que como já visto envolve as estratégias (apoio instrumental, emocional ou de informação) utilizadas pelo paciente para auxiliá-lo no enfrentamento frente ao tratamento oncológico, também apresentou correlação positiva com o domínio qualidade de vida total $(r=0,338)$, que está vinculado a uma percepção global do sujeito sobre sua $\mathrm{QV}$, incluindo facetas de cada um dos domínios (físico, psicológico, relações sociais e meio ambiente), indicando que a percepção de qualidade de vida juntamente com o meio social e o auxílio que este pode oferecer podem ser positivos aos pacientes.

Por meio da comparação dos resultados da presente pesquisa com dados apresentados por Souza e Araújo (2010) e Alegrance, Souza e Mazzei (2010), pode-se considerar que as correlações entre as estratégias de enfrentamento focalizadas no problema e a percepção de qualidade de vida, além de indicarem que estas estratégias interferem significativamente na qualidade de vida dos pacientes, mostram que esta relação pode gerar resultados adaptativos frente ao tratamento oncológico (Alegrance et al, 2010; Souza \& Araújo, 2010).Os dados obtidos por Souza e Araújo (2010), apontaram que após a intervenção as estratégias de enfrentamento apresentaram uma melhora significativa para todos os fatores da escala EMEP, assim como um aumento na percepção de qualidade de vida, além disso, houve uma redução nos sinais de ansiedade e depressão nos pacientes.

Já no trabalho de Alegrance et al (2010) a correlação entre os construtos percepção de qualidade de vida e estratégias de enfrentamento indicou que para as duas populações (mulheres com linfedema ou sem linfedema) a qualidade de vida geral é positiva assim como as estratégias de enfrentamento focalizadas no problema e a busca por suporte social, o que indica que as estratégias de enfrentamento utilizadas podem interferir na adaptação dessas pacientes refletindo diretamente sobre sua qualidade de vida.

Além disso, os dados apresentados no trabalho de Huguet et al (2009), indicaram que quando a amostra era comparada por estado civil, as pacientes que estavam em um relacionamento estável apresentaram resultados melhores tanto no domínio psicológico $(\mathrm{p}=0,04)$ quanto nas relações sociais $(\mathrm{p}=0,02)$, enquanto que no nível sócio econômico, as integrantes da classe A, B e C apresentaram resultados melhores que as da classe $\mathrm{D}$ e E para as dimensões física $(\mathrm{p}=0,01)$ e meio ambiente $(p=0,002)$. Por fim, de forma geral, as participantes do estudo apresentaram uma boa qualidade de vida, e os autores apontam que isso pode ser resultado do auxílio multidisciplinar oferecido pela instituição, além das variáveis socioeconômicas, de escolaridade, estado civil e conservação mamária.

Os resultados da amostra geral deste trabalho apontam que a população estudada, em sua maioria, apresenta um bom enfrentamento da situação e uma boa percepção de qualidade de vida, e isso, assim como no trabalho de $\mathrm{Hu}-$ guet et al (2009), pode ser reflexo do trabalho oferecido pela clínica onde realizam o tratamento e por sua equipe, além de outras variáveis não estudadas, como escolaridade, estado civil ou condição socioeconômica. Com base nesses resultados, e segundo Souza e Araújo (2010), pode-se concluir que os dados colaboram para o crescimento de pesquisas sobre o tema, além de reafirmar a importância 
da relação estratégias de enfrentamento e percepção de qualidade de vida, que, quando utilizadas de forma eficaz promove resultados significativos na vida do paciente e na sua adaptação frente à doença e ao tratamento.

Foi possível, com a construção e realização deste trabalho, atingir os objetivos propostos para o mesmo e concluir que a maior parte da população estudada faz um uso adequado das estratégias de enfrentamento focalizadas no problema e apresenta uma boa percepção sobre sua qualidade de vida, o que pode ser um fator importante na adaptação frente ao diagnóstico e ao tratamento oncológico. Além disso, foi reforçada a importância e relevância de trabalhos com essa temática, que ainda vem crescendo no meio científico devido à preocupação em ofertar apoio e suporte a essa população que mostra grande necessidade de suporte, acolhimento e escuta.

Essas necessidades, além de apontadas em literatura, puderam ser melhor observadas durante a coleta dos dados desta pesquisa, onde os participantes aproveitavam para compartilhar sua história de vida; suas vivências diante de um diagnóstico tão estigmatizado; diante de um tratamento que, na maioria das vezes, é cansativo, prolongado e cheio de efeitos colaterais; seus anseios, medos e angústias em relação a morte, a vida, ao prognóstico e ao tratamento; e também as esperanças, na maioria das vezes, de cura. Outro ponto que pode indicar a necessidade de escuta foi em relação à leitura dos instrumentos, onde a maioria dos pacientes solicitou que a pesquisadora fizesse a leitura de forma que a cada pergunta eles aproveitavam para externalizar seus sentimentos, pensamentos e histórias.

Ainda sobre essa experiência, pode ser observado durante o momento da coleta que os pacientes, na maioria das vezes, apresentam sentimentos de culpa em relação à doença. Além dessas necessidades e sentimentos observados, quase a totalidade da amostra pontuou que sentem falta de um espaço para verbalizar e entrar em contato com seus sentimentos, e que acreditam na importância do apoio psicológico tanto para eles quanto para seus familiares.

Diante disso, trabalhos como esse enriquecem e contribuem como conhecimento científico de forma que agrega e amplia as informações sobre a área, e também auxiliam profissionais da saúde, principalmente psicólogos, a traçarem estratégias terapêuticas e efetivas em sua prática, pois encontram um panorama das principais reações emocionais e dos aspectos psicológicos mais recorrentes nesses pacientes. Entretanto, considerando as condições da realização desse trabalho deparou-se com algumas limitações, pois a coleta foi realizada em uma amostra por conveniência em uma clínica particular, sendo assim, o suporte ofertado a esse paciente, desde a informação até o tratamento, é diferente se comparar as estruturas que o Sistema Único de Saúde pode oferecer, de forma que não se pode generalizar os resultados aqui alcançados.

\section{REFERÊNCIAS}

Alegrance, F. C., Souza, C. B., \& Mazzei, R. L. (2010). Qualidade de vida e estratégias de enfrentamento em mulheres com e sem linfedem após câncer de mama. Revista Brasileira de Cancerologia, 56(3), 341-351.

Baratto, C. C., Quintana, A. M., Monteiro, D. T., Wottrich, S. H., Domingues de Deus, M., \& Camargo, V. P. (2011). A psicologia no hospital: promoção da qualidade de vida em pacientes oncológicos. Anais da IV Jornada Cientifica em Psicologia - Desafios atuais nas práticas da psicologia (pp.98-109). Santa Cruz do Sul, RS.

Canciam, R. (2011). Psicossomática, psico-oncologia e câncer. Jundiaí, SP: Paco Editorial.

Carvalho, M. M. M. J. (Org.). (1994). Introdução à Psiconcologia. Campinas, SP: Editorial Psy.

Carvalho, V. A., Franco, M. H. P., Kovács, M. J., Liberato, R. P., Macieira, R. C., Veit, M. T., Gomes, M. J. B., \& Barros, L. H. C. (2008). Temas em Psico-oncologia. São Paulo: Summus Editorial.

Costa Neto, S. B. \&Araujo, T. C. C. F. (2008). Qualidade de vida do enfermo oncológico: Um panorama sobre o campo e suas formas de avaliação. Em V. A. Carvalho, M.H. P. Franco, M. J. Kovács, R. P. Liberato, R. C. Macieira, M. T. Veit, M. J. B.Gomes\& L. H. C. Barros (Orgs.), Temas em psico-oncologia (pp. 195-208). São Paulo: Summus.

Fleck, M. P. A. (2008). A avaliação de qualidade de vida-Guia para profissionais da saúde. Porto Alegre: Artmed.

Fleck, M. P. A, Leal, O. F., Louzada, S., Xavier, M.; Chachamovich, E., Vieira, G., Santos, L., \& Pinzon, V. (1999). Desenvolvimento da versão em português do instrumento de avaliação de qualidade de vida da Organização Mundial da Saúde (WHOQOL-100). Revista Brasileira de Psiquiatria, 2l(1), 19-28.

Gimenes, M. G. G. (1998). A pesquisa do enfrentamento na prática psico-oncológica. Em M. M. Carvalho (Org.), Resgatando o Viver: Psico-oncologia no Brasil (pp. 256).São Paulo: Summus.

Gimenes, M.G.G. \& Queiroz, B. (1997). As diferentes fases de enfrentamento durante o primeiro ano após a mastectomia. Em M.G.G. Gimenes \& M.H. Fávero (Orgs). A mulher e o câncer (pp. 171-195). Campinas: Editorial Psy.

Huguet, P. R., Morais, S. S., Osis, M. J. D., Pinto-neto, A. M., \& Gurgel, M. S. C. (2009). Qualidade de vida e sexualidade de mulheres tratadas de câncer de mama. Revista Brasileira de Ginecologia e Obstetrícia, 31(2), 61-67.

INCA - Instituto Nacional de Câncer José de Alencar Gomes da Silva (2012). Estimativa 2012 - Incidência de câncer no Brasil. Disponível em: http://www.inca.gov.br/estimativa/2012/. Acesso em 20/09/2012. 
Lorencetti, A. \& Simonetti, J. P. (2005). As estratégias de enfrentamento de pacientes durante o tratamento de radioterapia. Revista Latino-americana de Enfermagem,13(6), 944-950.

Pestana, J. P., Estevens, D. \&Conboy, J. (2007). O papel da espiritualidade na qualidade de vida do doente oncológico em quimioterapia. Psicologia.com.pt - O Portal dos Psicólogos. Retirado em 11/10/2008, de http://www.psicologia.com.pt/ publicar/citar.php.

Roque V. M. N. \& Forones N.M. (2006). Avaliação da qualidade de vida e toxicidades em pacientes com câncer colorretal tratados com quimioterapia adjuvante baseada em fluoropirimidinas. Arquivos Gastroenterologia, 43(2).

Santana, J. J. R. A., Zanin, C. R., \& Maniglia, J. V. (2008). Pacientes com câncer: enfrentamento, rede social e apoio social. Paidéia, 18(40), 371-384.
Sebben, R. (2007). Psiconcologia: o paciente e a família frente ao diagnostico e tratamento do câncer. Universidade Vale do Itajaí Centro de educação de ciências da saúde. Itajaí, SC.

Seidl, E. M. F., Tróccoli, B. T. \& Zannon, C. M. L. C. (2001). Análise fatorial de uma medida de estratégias de enfrentamento. Psicologia: teoria e pesquisa, 17(3), 225-234.

Souza, J. R. (2009). Estudo sobre avaliação de eficácia terapêutica em oncologia: grupo psicoeducacional "Aprendendo a Enfrentar”. Dissertação apresentada ao Instituto de Psicologia da Universidade de Brasília, Brasília, DF.

Souza, J. R., \& Araújo, T. C. C. F. (2010). Eficácia terapêutica de intervenção em grupo psicoeducacional: um estudo exploratório em oncologia. Estudos de Psicologia, 27(2),187-196. 